\title{
Electronic instabilities of a Hubbard model approached as a large array of coupled chains: Competition between $d$-wave superconductivity and pseudogap phase
}

\author{
E. Perfetto ${ }^{1,2}$ and J. González ${ }^{1}$ \\ ${ }^{1}$ Instituto de Estructura de la Materia, Consejo Superior de Investigaciones Científicas, Serrano 123, 28006 Madrid, Spain \\ ${ }^{2}$ Consorzio Nazionale Interuniversitario per le Scienze Fisiche della Materia, Universitá di Roma Tor Vergata, Via della Ricerca \\ Scientifica 1, 00133 Roma, Italy \\ (Received 9 January 2007; revised manuscript received 28 June 2007; published 12 February 2008)
}

\begin{abstract}
We study the electronic instabilities in a two-dimensional (2D) Hubbard model where one of the dimensions has a finite width, so that it can be considered as a large array of coupled chains. The finite transverse size of the system gives rise to a discrete string of Fermi points, with respective electron fields that, due to their mutual interaction, acquire anomalous scaling dimensions depending on the point of the string. Using bosonization methods, we show that the anomalous scaling dimensions vanish when the number of coupled chains goes to infinity, implying the Fermi liquid behavior of a $2 \mathrm{D}$ system in that limit. However, when the Fermi level is at the Van Hove singularity arising from the saddle points of the 2D dispersion, backscattering and Cooper-pair scattering lead to the breakdown of the metallic behavior at low energies. These interactions are taken into account through their renormalization group scaling, studying in turn their influence on the nonperturbative bosonization of the model. We show that, at a certain low-energy scale, the anomalous electron dimension diverges at the Fermi points closer to the saddle points of the 2D dispersion. The $d$-wave superconducting correlations also become large at low energies, but their growth is cut off as the suppression of fermion excitations takes place first, extending progressively along the Fermi points toward the diagonals of the 2D Brillouin zone. We stress that this effect arises from the vanishing of the charge stiffness at the Fermi points, characterizing a critical behavior that is well captured within our nonperturbative approach.
\end{abstract}

DOI: 10.1103/PhysRevB.77.054504

PACS number(s): 74.72.-h, 71.10.Hf, 71.10.Fd, 71.10.Pm

\section{INTRODUCTION}

Copper-oxide superconductors have shown, since their discovery, quite unusual electronic properties regarding the particular features of the superconducting state as well as the behavior of the normal state. One of the most remarkable experimental observations has been the existence of the socalled pseudogap at temperatures which are above the superconducting dome in the phase diagram. ${ }^{1}$ The unconventional character of this pseudogap lies in the fact that it opens up quite anisotropically along the Fermi line of the electron system. At some doping-dependent temperature $T^{*}$, which is, in general, well above the transition temperature of the superconducting phase, electron quasiparticles become suppressed in the region of the Fermi line which is closer to the momenta $(\pi, 0)$ and $(0, \pi)$. This is particularly manifest in the angle-resolved photoemission spectroscopy experiments, ${ }^{2}$ as the quasiparticle peaks get a dispersion that does not cross the Fermi level at any point in momentum space. As the temperature is lowered from $T^{*}$, this partial destruction of the Fermi line takes place progressively over wider regions, approaching the diagonals of the Brillouin zone. ${ }^{3}$

There have been several attempts to provide a theoretical explanation for the pseudogap in the cuprates. Some of the proposals have considered the possible coupling of the electron quasiparticles to collective boson excitations. ${ }^{4,5}$ It has also been investigated the possibility that the electron system may develop an ordered phase, which would induce the appearance of a gap in the single-particle spectrum. ${ }^{6,7}$ Other explanations are based on the strong electron correlations and, in particular, on the description of the cuprates in terms of the Hubbard model. Thus, it has been proposed that theo- ries based on a resonating-valence-bond state ${ }^{8}$ or on the existence of states with time-reversal symmetry-breaking ${ }^{9}$ may give rise to features consistent with the pseudogap phase.

The main challenge that face all these proposals is actually the difficulty of dealing with a regime of strong electron correlations. In these conditions, one usually has to resort to mean-field approximations that may give a qualitative idea of the possible phases of the electron system. It would also be desirable to have an alternative framework in which the nature of the pseudogap could be clarified. In this regard, the motivation of the present paper is to investigate the Hubbard model by means of a nonperturbative approach, trying to capture the effects of the strong electron correlations. For that purpose, we will consider a two-dimensional (2D) model where one of the dimensions has a finite width, so that it may be considered as formed by the coupling of a large number of Hubbard chains. A general analysis of the $N$-chain Hubbard model in weak coupling has been accomplished in Ref. 10. We will use bosonization techniques to determine the nonperturbative effects of the interactions with small momentum transfer on the properties of the fermion excitations. In this framework, the Fermi liquid properties of a 2D system are recovered when the number of coupled chains goes to infinity, as the anomalous scaling dimensions of the electron fields vanish in that limit. The description has to be completed anyhow by considering the interactions whose momentum transfer is not small. These will be taken into account through their renormalization group scaling, studying their influence on the nonperturbative bosonization of the model and the instabilities they may trigger at low energies.

The most interesting instance corresponds to the case where the Fermi level is near the Van Hove singularity aris- 
ing from the saddle points in the 2D dispersion at $(\pi, 0)$ and $(0, \pi)$. When the $2 \mathrm{D}$ model is approached by a large array of coupled chains, the fermion excitations are attached to a large but finite number of Fermi points, with respective electron fields that are renormalized by the interactions. We will see that, for the model with on-site repulsion, the angleresolved quasiparticle weight vanishes below a certain lowenergy scale, starting from the Fermi points which are closer to $(\pi, 0)$ and $(0, \pi)$. The $d$-wave superconducting correlations are also dominant at low energies, but their growth is cut off as the suppression of fermion excitations takes place first, extending progressively along the Fermi points toward the diagonal of the Brillouin zone.

The results that we present are in part reminiscent of those obtained in a $2 \mathrm{D}$ electron system when the Fermi line is placed close to the saddle points at $(\pi, 0)$ and $(0, \pi) \cdot{ }^{11-23}$ It has been shown that the presence of a Van Hove singularity induces a strong renormalization of the quasiparticle properties, with a clear suppression of the quasiparticle weight near the saddle points. ${ }^{15,23}$ Some studies have also remarked that the divergent flow of the couplings observed in renormalization group analyses could lead to the vanishing of both the compressibility and the magnetic susceptibility. ${ }^{17,19,20}$ In our approach, we are able to identify the onset of the destruction of the Fermi line at an energy scale where the electron correlations have not entered yet the divergent regime. This is possible as long as the Fermi surface instability appears as a critical point in the nonperturbative quasiparticle renormalization arising from the bosonization approach. The present combination of bosonization and renormalization group methods may be seen as an alternative to conventional analyses of 2D electron systems near the Van Hove filling, offering a sensible $2 \mathrm{D}$ limit as the number of coupled chains is increased.

\section{QUASIPARTICLE PROPERTIES IN THE LIMIT OF LARGE NUMBER OF CHAINS}

We will take as our starting point a $2 \mathrm{D}$ model with nearest-neighbor and next-to-nearest-neighbor hoppings, with a finite size in one of the spatial dimensions. For the time being, we introduce a generic spin-independent interaction with potential $V$. The Hamiltonian of the system is then given by

$$
\begin{aligned}
H= & \sum_{j=1}^{N} \sum_{n=-\infty}^{\infty}\left[-t \psi_{j \sigma}^{\dagger}(n) \psi_{j \sigma}(n+1)-t \psi_{j \sigma}^{\dagger}(n) \psi_{j+1 \sigma}(n)\right. \\
& \left.-t^{\prime} \psi_{j \sigma}^{\dagger}(n) \psi_{j+1 \sigma}(n \pm 1)+\text { H.c. }\right] \\
& +\sum_{j, l=1}^{N} \sum_{m, n=-\infty}^{\infty} \psi_{j \sigma}^{\dagger}(m) \psi_{j \sigma}(m) V_{j l}(m, n) \psi_{l \sigma^{\prime}}^{\dagger}(n) \psi_{l \sigma^{\prime}}(n),
\end{aligned}
$$

where $\psi_{j \sigma}^{\dagger}(n)$ and $\psi_{j \sigma}(n)$ are electron creation and annihilation operators at site $n$ of the $j$ th chain, with spin projection given by the $\sigma$ index.

For the description of quasiparticle properties, it is convenient to make the passage to electron operators in momentum

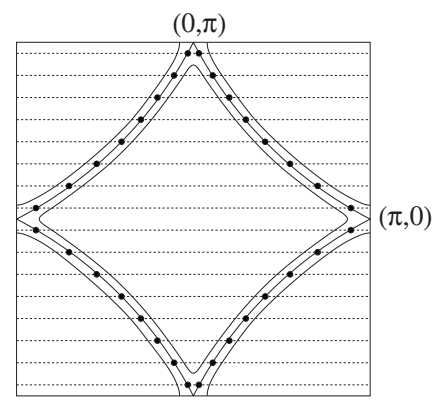

FIG. 1. Schematic representation of the Fermi points for a 2D model made of a finite number of coupled chains, when the Fermi level is at the Van Hove singularity. The horizontal lines correspond to quantized transverse momenta for twisted boundary conditions, $k_{a}=\pi(2 a+1) / N$, with $a=-N / 2,-N / 2+1, \ldots, N / 2-1$. Energy contour lines are also represented for the cutoff at $E_{c}$ and $-E_{c}$ about the Fermi level.

space, taking the Fourier transform in the site and chain variables:

$$
\psi_{j \sigma}(n)=\frac{1}{\sqrt{N}} \sum_{a} \int_{-\pi}^{\pi} d p e^{-i k_{a} j} e^{-i p n} \psi_{\sigma}\left(k_{a}, p\right) .
$$

We will apply periodic boundary conditions to the system of $N$ chains. Whenever it is convenient to exclude the singular points $(\pi, 0)$ and $(0, \pi)$ from the spectrum, we will make, however, a twist by $e^{i \pi}$ in the electron field after completing a whole period along the array. With these twisted boundary conditions, the transverse momentum $k_{a}$ will be quantized according to the rule $k_{a}=\pi(2 a+1) / N, \quad a=-N / 2,-N / 2$ $+1, \ldots, N / 2-1$. From the Hamiltonian (1), we get the dispersion relation for the different transverse momenta

$$
\varepsilon_{a}(p)=-2 t \cos (p)-2 t \cos \left(k_{a}\right)-4 t^{\prime} \cos (p) \cos \left(k_{a}\right) .
$$

Furthermore, for the sake of describing low-energy properties, we may concentrate on the modes that are within a cutoff energy $E_{c}$ about the Fermi energy $\varepsilon_{F}$, where the dispersion becomes approximately linear about the Fermi points. This is illustrated in Fig. 1. For each transverse momentum $k_{a}$, the low-energy excitations can be encoded into two fields $\psi_{a \sigma}(p)$ and $\psi_{\bar{a} \sigma}(p)$ with opposite chirality (i.e., right- or left-moving character), corresponding to the two different Fermi points at $p_{a}$ and $-p_{a}$ :

$$
\begin{gathered}
\int d p \psi_{\sigma}^{\dagger}\left(k_{a}, p\right)\left[\varepsilon_{a}(p)-\varepsilon_{F}\right] \psi_{\sigma}\left(k_{a}, p\right) \\
\approx \int d p v_{a}\left[\psi_{a \sigma}^{\dagger}(p)\left(p-p_{a}\right) \psi_{a \sigma}(p)\right. \\
\left.+\psi_{\bar{a} \sigma}^{\dagger}(p)\left(-p-p_{a}\right) \psi_{\bar{a} \sigma}(p)\right],
\end{gathered}
$$

$v_{a}$ being the Fermi velocity at $p_{a}$ and $-p_{a}$.

From the detailed discussion in Ref. 10, it follows that $E_{c}$ can be taken suitably small so that the possible scattering processes are severely limited by momentum conservation. The price that one has to pay for the reduction of the cutoff is that the original interactions are corrected by terms which 


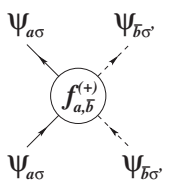

(a)

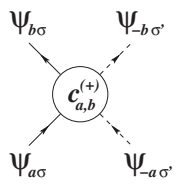

(c)

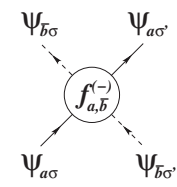

(b)

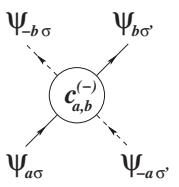

(d)
FIG. 2. Interaction processes that survive at low energies within the cutoff $E_{c}$ about the Fermi level. The solid and dashed lines correspond to the two different chiralities (right- and left-moving character) of the electron fields.

are of the order of $\sim(V / 2 \pi t)^{2} \log \left(k_{F} / E_{c}\right), k_{F}$ being the scale of the size of the Fermi line. In our model, we will start with an on-site interaction $U$ such that $U / 2 \pi t<1$, in such a way that the perturbative corrections are under control down to a conveniently small cutoff $E_{c}$.

Confining our discussion to the low-energy modes in the interval between $\varepsilon_{F}-E_{c}$ and $\varepsilon_{F}+E_{c}$, there is a reduced number of ways in which the momentum can be conserved in the scattering processes. These can be classified into four different interaction channels, as represented in Fig. 2. We may have processes with suitably small momentum transfer between interacting particles near Fermi points $a$ and $b$ (to which we will assign couplings $\left.f_{a, b}^{(+)}\right)$and processes where the particles exchange their positions in the neighborhood of the two Fermi points (which we will label with couplings $f_{a, b}^{(-)}$). The other type of interactions that exist generically at low energies corresponds to processes where the total momentum of the incoming particles is almost vanishing. In this case, we can discern a channel where the particles interact keeping their respective chiralities (with couplings that we will denote by $c_{a, b}^{(+)}$) and another where they exchange their chiralities (requiring then different couplings $\left.c_{a, b}^{(-)}\right) .{ }^{24}$ The Hamiltonian involving the low-energy excitations then becomes

$$
\begin{aligned}
H= & \sum_{a} \int d p v_{a}\left[\psi_{a \sigma}^{\dagger}(p)\left(p-p_{a}\right) \psi_{a \sigma}(p)+\psi_{\bar{a} \sigma}^{\dagger}(p)\right. \\
& \left.\times\left(-p-p_{a}\right) \psi_{\bar{a} \sigma}(p)\right]+\sum_{\langle a, b\rangle} \int d p d p^{\prime} d q \\
& \times\left\{\left[\psi_{a \sigma}^{\dagger}(p+q) \psi_{a \sigma}(p) f_{a, b}^{(+)} \psi_{b \sigma^{\prime}}^{\dagger}\left(p^{\prime}-q\right) \psi_{b \sigma^{\prime}}\left(p^{\prime}\right)\right.\right. \\
& +\psi_{b \sigma}^{\dagger}(p+q) \psi_{a \sigma}(p) f_{a, b}^{(-)} \psi_{a \sigma^{\prime}}^{\dagger}\left(p^{\prime}-q\right) \psi_{b \sigma^{\prime}}\left(p^{\prime}\right) \\
& +\psi_{a \sigma}^{\dagger}(p+q) \psi_{a \sigma}(p) f_{a, \bar{b}}^{(+)} \psi_{\bar{b} \sigma^{\prime}}^{\dagger}\left(p^{\prime}-q\right) \psi_{\bar{b} \sigma^{\prime}}\left(p^{\prime}\right) \\
& +\psi_{\bar{b} \sigma}^{\dagger}(p+q) \psi_{a \sigma}(p) f_{a, b}^{(-)} \psi_{a \sigma^{\prime}}^{\dagger}\left(p^{\prime}-q\right) \psi_{\bar{b} \sigma^{\prime}}\left(p^{\prime}\right) \\
& +a \leftrightarrow \bar{a}, b \leftrightarrow \bar{b}]+\psi_{b \sigma}^{\dagger}(p+q) \psi_{a \sigma}(p) c_{a, b}^{(+)} \psi_{-b \sigma^{\prime}}^{\dagger}\left(p^{\prime}-q\right)
\end{aligned}
$$

$$
\begin{aligned}
& \times \psi_{-a \sigma^{\prime}}\left(p^{\prime}\right)+\psi_{-b \sigma}^{\dagger}(p+q) \psi_{a \sigma}(p) c_{a, b}^{(-)} \psi_{b \sigma^{\prime}}^{\dagger}\left(p^{\prime}-q\right) \\
& \left.\times \psi_{-a \sigma^{\prime}}\left(p^{\prime}\right)\right\}
\end{aligned}
$$

where the indices in the sum over pairs $\langle a, b\rangle$ run over just one of the chiralities.

To clarify our notation, we recall that the index $\bar{a}$ corresponds to a Fermi point with the same transverse momentum but opposite chirality to that represented by the index $a$. Thus, the difference between the interactions $f_{a, b}^{(+)}$and $f_{a, b}^{(+)}$is that the first involves modes which have the same chirality, while $f_{a, \bar{b}}^{(+)}$couples an electron current made of right-moving fields to another current of left-moving fields. On the other hand, the difference between the interactions represented by $f_{a, \bar{b}}^{(+)}$and $f_{a, \bar{b}}^{(-)}$is that, in the latter, the incoming particles exchange their chirality in the scattering process. We finally remark that, in our notation, the index $-a$ represents a Fermi point with a momentum completely opposite to that of Fermi point $a$ (so that the modes around Fermi point $-a$ have opposite chirality to those around Fermi point $a$ ). In this way, the incoming particles are bound to have very small total momentum in the interactions represented by $c_{a, b}^{(+)}$and $c_{a, b}^{(-)}{ }^{25}$

An important difference between the interactions of particles with like chirality $\left(f_{a, b}^{(+)}, f_{\bar{a}, \bar{b}}^{(+)}, f_{a, b}^{(-)}\right.$, and $\left.f_{\bar{a}, \bar{b}}^{(-)}\right)$and those corresponding to $f_{a, \bar{b}}^{(+)}, f_{a, \bar{b}}^{(-)}, c_{a, b}^{(+)}$, and $c_{a, b}^{(-)}$is that the strength of the latter depends on the energy scale. This may induce instabilities that appear when the system is observed at scales very close to the Fermi level. On the other hand, the significance of the interactions with small momentum transfer $\left(f_{a, b}^{(+)}\right.$, $f_{\bar{a}, \bar{b}}^{(+)}, f_{a, \bar{b}}^{(+)}$, and $\left.f_{\bar{a}, b}^{(+)}\right)$is that they dictate the class of electron liquid describing the normal state (metallic) properties of the coupled chains. This corresponds to the so-called Luttinger liquid for any finite number of chains. ${ }^{26}$ We discuss next the way in which the limit of a large number of chains may change the quasiparticle properties, depending on the given Fermi point.

The Luttinger liquid properties are best described in terms of operators given by the density fluctuations at small momentum:

$$
\begin{aligned}
& B_{a}^{\dagger}(q)=\frac{1}{\sqrt{|q|}} \int d p \psi_{a \sigma}^{\dagger}(p+q) \psi_{a \sigma}(p), \quad q>0, \\
& B_{\bar{a}}^{\dagger}(q)=\frac{1}{\sqrt{|q|}} \int d p \psi_{\bar{a} \sigma}^{\dagger}(p+q) \psi_{\bar{a} \sigma}(p), \quad q<0 .
\end{aligned}
$$

It is well known that the kinetic part of the Hamiltonian (5) can be written in terms of the operators (6) and (7). ${ }^{26}$ For the time being, we will restrict the discussion to the interactions preserving the chirality of the particles. Then, we end up with the hamiltonian governing the Luttinger liquid dynamics 


$$
\begin{aligned}
H_{L L}= & \sum_{a} v_{a}\left(\int_{q>0} d q|q| B_{a}^{\dagger}(q) B_{a}(q)+\int_{q<0} d q|q| B_{\bar{a}}^{\dagger}(q) B_{\bar{a}}(q)\right) \\
& +\sum_{\langle a, b\rangle} \int_{q>0} d q|q|\left\{f_{a, b}^{(+)}\left[B_{a}^{\dagger}(q) B_{b}(q)+B_{a}(q) B_{b}^{\dagger}(q)\right]\right. \\
& \left.+f_{a, \bar{b}}^{(+)}\left[B_{a}^{\dagger}(q) B_{\bar{b}}^{\dagger}(-q)+B_{a}(q) B_{\bar{b}}^{-}(-q)\right]\right\} \\
& +\sum_{\langle a, b\rangle} \int_{q<0} d q|q|\left\{f_{\bar{a}, \bar{b}}^{(+)}\left[B_{\bar{a}}^{\dagger}(q) B_{\bar{b}}(q)+B_{\bar{a}}(q) B_{\bar{b}}^{\dagger}(q)\right]\right. \\
& \left.+f_{\bar{a}, b}^{(+)}\left[B_{\bar{a}}^{\dagger}(q) B_{b}^{\dagger}(-q)+B_{\bar{a}}(q) B_{b}(-q)\right]\right\}
\end{aligned}
$$

The Hamiltonian $H_{L L}$ can be diagonalized by making the change of variables

$$
\begin{gathered}
\widetilde{B}_{a}^{\dagger}(q)=\sum_{b}\left[s_{a b} B_{b}^{\dagger}(q)+t_{a \bar{b}} B_{\bar{b}}(-q)\right], \\
\widetilde{B}_{\bar{a}}(-q)=\sum_{b}\left[s_{\bar{a} \bar{b}} B_{\bar{b}}(-q)+t_{\bar{a} b} B_{b}^{\dagger}(q)\right] .
\end{gathered}
$$

The change of basis defined by the matrix elements $s_{a b}, t_{a b}$ is not unitary, since the requirement of canonical commutation relations for the new operators imply, in particular, that

$$
\sum_{b}\left(\left|s_{a b}\right|^{2}-\left|t_{a b}\right|^{2}\right)=1 .
$$

For the sake of carrying out the diagonalization of $H_{L L}$ with great computational efficiency, one can introduce, however, the following trick. By redefining all the boson operators with left-handed character, for instance,

$$
B_{\bar{a}}(q)=i \hat{B}_{\bar{a}}(q), \quad B_{\bar{a}}^{\dagger}(q)=i \hat{B}_{\bar{a}}^{\dagger}(q),
$$

we change the canonical commutation relations to

$$
\left[\hat{B}_{\bar{a}}(q), \hat{B}_{\bar{a}}^{\dagger}\left(q^{\prime}\right)\right]=-\delta\left(q-q^{\prime}\right) .
$$

By keeping $B_{a}(q)=\hat{B}_{a}(q)$ and $B_{a}^{\dagger}(q)=\hat{B}_{a}^{\dagger}(q)$ for the other chirality, the linear change of variables for the redefined operators has matrix elements that correspond to a unitary change of basis. Thus, by performing first the redefinition (12), one can apply then standard algorithms that are able to diagonalize very large complex matrices. By reverting at the end the transformation (12), it is possible then to bring the Hamiltonian (8) to the form

$H_{L L}=\sum_{a} \widetilde{v}_{a}\left(\int_{q>0} d q|q| \widetilde{B}_{a}^{\dagger}(q) \widetilde{B}_{a}(q)+\int_{q<0} d q|q| \widetilde{B}_{\bar{a}}^{\dagger}(q) \widetilde{B}_{\bar{a}}(q)\right)$.

Our aim is to show the evolution of the quasiparticle properties at the hot spots around the saddle points $(\pi, 0)$ and $(0, \pi)$. As a most relevant instance, we give the results of performing the diagonalization of the Hamiltonian (8) for a growing number of coupled chains with $t^{\prime}=-0.2 t$, at the filling that makes the 2D Fermi line go across the saddle points. We have taken an on-site interaction $U$, that leads to a set of momentum-independent couplings equal to $U / N$ in the
Hamiltonian (5). In general, we may interpret that the original boson operators are dressed by the interactions. The different correlation functions can be obtained by expressing the operators $B_{a}^{\dagger}(q)$ and $B_{a}(q)$ in terms of the set of noninteracting operators in Eq. (14), in such a way that the interaction effects are encoded in the string $\left\{\widetilde{v}_{a}\right\}$ and in the parameters of the transformation (10).

The most important piece of information comes, after the diagonalization, from the change of basis that brings the Hamiltonian to the form (14). The original boson operators can be obtained from the free operators that appear in Eq. (14) through the inverse change of variables

$$
\begin{gathered}
B_{a}^{\dagger}(q)=\sum_{b}\left[\widetilde{s}_{a b} \widetilde{B}_{b}^{\dagger}(q)+\widetilde{t}_{a \bar{b}} \widetilde{B}_{\bar{b}}(-q)\right], \\
B_{\bar{a}}(-q)=\sum_{b}\left[\widetilde{s}_{\bar{a} \bar{b}} \widetilde{B}_{\bar{b}}(-q)+\widetilde{t}_{\bar{a} b} \widetilde{B}_{b}^{\dagger}(q)\right] .
\end{gathered}
$$

In the absence of interaction, the only nonvanishing coefficients are the diagonal elements $\widetilde{s}_{a a}=\widetilde{s}_{\bar{a} \bar{a}}=1$. The interactions give rise, however, to anomalous scaling dimensions $\gamma_{a}$ of the electron fields, which can be obtained from the spatial decay of the electron propagator at equal time $t$

$$
\left\langle\psi_{a \sigma}^{\dagger}(x, t) \psi_{a \sigma}(y, t)\right\rangle \sim \frac{1}{|x-y|^{1+\gamma_{a}}} .
$$

It is well known that the fermion fields $\psi_{a \sigma}, \psi_{\bar{a} \sigma}$ are recovered by exponentiation of the integral of the respective chiral density operators $\rho_{a}(x), \rho_{\bar{a}}(x) .{ }^{26}$ These are given according to Eqs. (6) and (7) by

$$
\rho_{a}(x)=\int_{q>0} d q \sqrt{|q|} e^{-i q x} B_{a}^{\dagger}(q)+\int_{q<0} d q \sqrt{|q|} e^{-i q x} B_{a}(-q),
$$

$$
\rho_{\bar{a}}(x)=\int_{q>0} d q \sqrt{|q|} e^{-i q x} B_{\bar{a}}(-q)+\int_{q<0} d q \sqrt{|q|} e^{-i q x} B_{\bar{a}}^{\dagger}(q) .
$$

Then, it can be seen that the charge sector contributes to the exponent in Eq. (17) by

$$
\frac{1}{2}+\gamma_{a}=\frac{1}{2} \sum_{b}\left(\left|\widetilde{s}_{a b}\right|^{2}+\left|\widetilde{t}_{a \bar{b}}\right|^{2}\right)
$$

We observe that, as soon as the interaction is switched on, we get an anomalous dimension $\gamma_{a} \neq 0$, reflecting the disappearance of the quasiparticle pole in the electron propagator.

It is very instructive to observe the behavior of the anomalous dimension $\gamma_{a}$ at different Fermi points of the model. We find that, away from $(\pi, 0)$ and $(0, \pi)$, the anomalous dimension $\gamma_{a}$ decreases in the limit of large number $N$ of chains, following with great accuracy a $1 / N$ behavior. ${ }^{27}$ The prefactor for this law depends, however, on the distance to the saddle points, and it grows as one gets closer to $(\pi, 0)$ or $(0, \pi)$, as shown in Fig. 3. The limit toward these points becomes actually quite singular. If one takes the limit of large $N$ sitting at the Fermi point which is away but nearest 


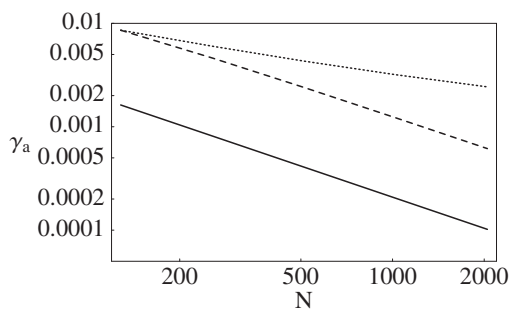

FIG. 3. Dependence of the anomalous electron dimension $\gamma_{a}$ on the number $N$ of coupled chains in a Hubbard model with periodic boundary conditions, when the Fermi point is taken close to the diagonal of the Brillouin zone (full line), with a constant transverse momentum $k_{a}=\pi / 64$ (dashed line), and at the nearest location to the saddle point with $k_{a}=2 \pi / N$ (dotted line).

to the saddle point (so that its Fermi velocity decreases consequently as $1 / N)$, we observe that the anomalous dimension also decreases monotonically, but with a behavior that seems to be smoother than any power law, as observed from Fig. 3. The sizes for which we have been able to diagonalize the model (up to $\approx 2000$ chains) do not allow us to assure whether this limit to the saddle point may produce a vanishing anomalous dimension as $N \rightarrow \infty$. Our results make clear anyhow that, for a Fermi point placed at any finite distance from the saddle points, the anomalous dimension vanishes in the limit of very large number of chains. This is what allows us to take the limit $N \rightarrow \infty$ as a way of approaching the properties of an interacting Fermi liquid in two dimensions. We will see actually that the presence of backscattering and Cooper-pair scattering modifies significantly the present picture, leading to an unconventional low-energy behavior near the hot spots for a large number of Fermi points.

\section{RENORMALIZATION GROUP APPROACH TO LOW- ENERGY PHASES}

In order to describe the quasiparticle properties at low energies, one has to incorporate the effects of the variation of the couplings $f_{a, \bar{b}}^{(+)}, f_{a, b}^{(-)}, c_{a, b}^{(+)}$, and $c_{a, b}^{(-)}$with the energy scale. The discussion of the preceding section is pertinent at the energy scale $E_{c}$, where the dispersion becomes approximately linear about the Fermi level. At lower energy scales, the effective values of the couplings may change significantly, having a large impact on the renormalization of the quasiparticle properties.

We have to remark at this point that the renormalization effects manifest, in general, not only in the quasiparticle parameters, but also give rise to changes in the shape of the Fermi surface. It has been proposed that, within a renormalization group approach at constant particle number, the Fermi surface has to be understood as a fixed point of the renormalization group. ${ }^{28}$ A practical implementation of this idea for a two-chain model can be found in Ref. 29. In the present context, to find such a fixed-point solution is beyond reach for the large string of Fermi points, as the Fermi surface is reshaped also by irrelevant interactions whose momenta are not constrained by the cutoff and that proliferate at large $N$ even more rapidly than the couplings already consid- ered. Being interested in the situation where the Fermi level is close to a Van Hove singularity, it will be enough for us to guarantee that the Fermi line is not driven away from the saddle points at $(\pi, 0)$ and $(0, \pi)$ upon switching the interaction. We rely for that on the evidence obtained with the renormalization group at fixed chemical potential, which provides a sensible approach to systems in contact with a large reservoir of particles. In this context, it has been actually shown that the Van Hove singularity acts as an attractor, pinning the Fermi level for a certain window in the choice of the chemical potential. ${ }^{12,15}$ We motivate in this way the suitability of studying the model with the Fermi line near $(\pi, 0)$ and $(0, \pi)$, as this choice does not imply the fine-tuning of the chemical potential in the system (as fixed, for instance, by the contact with a charge reservoir).

Below the scale $E_{c}$, the four-fermion interaction vertices are corrected by diagrams that depend logarithmically on the energy scale $\Lambda$ (measured from the Fermi energy $\varepsilon_{F}$ ). To deal with this divergence, one can integrate progressively the electron modes starting from the limits of the linear branches at $\varepsilon_{F} \pm E_{c}{ }^{30}$ This leads to a dependence of the couplings on the scale $l=\log \left(E_{c} / \Lambda\right)$, governed by scaling equations that read at the one-loop level

$$
\begin{gathered}
\frac{\partial f_{a, \bar{b}}^{(+)}}{\partial l}=-\frac{1}{2 \pi v_{a b}}\left[\left(f_{a, \bar{b}}^{(-)}\right)^{2}-\left(c_{a,-b}^{(+)}\right)^{2}\right] \\
\frac{\partial f_{a, \bar{b}}^{(-)}}{\partial l}=-\frac{1}{\pi v_{a b}}\left[\left(f_{a, b}^{(-)}\right)^{2}+\left(c_{a,-b}^{(-)}\right)^{2}-c_{a,-b}^{(-)} c_{a,-b}^{(+)}\right], \\
\frac{\partial c_{a, b}^{(+)}}{\partial l}=-\sum_{c} \frac{1}{2 \pi v_{c}}\left(c_{a, c}^{(+)} c_{c, b}^{(+)}+c_{a, c}^{(-)} c_{c, b}^{(-)}\right)+\frac{1}{2 \pi v_{a b}} c_{a, b}^{(+)} h_{a,-b}^{(+)}, \\
\frac{\partial c_{a, b}^{(-)}}{\partial l}=-\sum_{c} \frac{1}{2 \pi v_{c}}\left(c_{a, c}^{(+)} c_{c, b}^{(-)}+c_{a, c}^{(-)} c_{c, b}^{(+)}\right)-\frac{1}{\pi v_{a b}} c_{a, b}^{(-)} h_{a,-b}^{(-)} \\
-\frac{1}{2 \pi v_{a b}} \sum_{s} c_{a, b}^{(s)} h_{a,-b}^{(-s)},
\end{gathered}
$$

where $h_{a,-b}^{(s)} \equiv 2 f_{a,-b}^{(s)}-\delta_{a b} c_{a, a}^{(s)}$, and the $v_{a b}$ are defined in terms of the Fermi velocities as $v_{a b}=\left(v_{a}+v_{b}\right) / 2 .^{31}$

We have solved numerically the renormalization group equations for a Hubbard model with next-to-nearestneighbor hopping $t^{\prime}=-0.2 t$. Our main interest focuses on the instabilities arising from the hot spots around the momenta $(\pi, 0)$ and $(0, \pi)$. Thus, we have chosen the filling to match the Fermi level with the position of the Van Hove singularity from the saddle points. We have considered systems with up to $N=76$ and 152 Fermi points, which already demand the introduction of $\approx 5900$ independent couplings. We have assumed that the system becomes periodic in the transverse direction, but using twisted boundary conditions to avoid the presence of Fermi points with vanishing Fermi velocity at $(\pi, 0)$ and $(0, \pi)$. Anyhow, in this approach we are able to capture the physics of the hot spots by increasing the number 
$N$, since the lowest Fermi velocity (corresponding to $k_{a}$ $=\pi / N$ and $\left.k_{a}=\pi-\pi / N\right)$ scales as $2 \pi\left(t^{2}-4 t^{\prime 2}\right)^{1 / 2} / N$. It is worthy to point out that the model remains exactly $C_{4 v}$ symmetric with the mentioned choice of filling level.

We have started the integration of the equations in the weak-coupling regime, taking a value of the on-site Hubbard repulsion $U=t$. We recall that, when making the passage to the electron fields in momentum space, all the couplings in the Hamiltonian (5) get a contribution equal to $U / N$ from the on-site interaction. In any event, the flow is driven to a regime where some of the couplings grow large. In particular, at some energy scale $\Lambda^{*}=E_{c} e^{-l^{*}}$, a divergence is found in some of the couplings. For $N=76$, for instance, we find that $l^{*}=4.99$. As observed in Ref. 10, the singularity corresponds to the divergence of interactions involving scattering between the Fermi points with smallest velocity, i.e., those nearest to the hot spots. The rest of the couplings remain in the weak-coupling regime.

In order to characterize the instability from the large growth of the couplings, we have also computed the scaling of the response functions $R^{(\eta)}(\omega)$ for singlet (SS) and triplet superconducting (TS) order parameters, as well as for charge-density-wave (CDW) and spin-density-wave (SDW) order parameters. The superconducting order parameters, for instance, are given by

$$
\begin{aligned}
& O_{a}^{(S S)}=\int d p\left[\psi_{a \uparrow}(p) \psi_{-a \downarrow}(-p)-\psi_{a \downarrow}(p) \psi_{-a \uparrow}(-p)\right], \\
& O_{a}^{(T S)}=\int d p\left[\psi_{a \uparrow}(p) \psi_{-a \downarrow}(-p)+\psi_{a \downarrow}(p) \psi_{-a \uparrow}(-p)\right] .
\end{aligned}
$$

These operators build response function matrices defined in the space of Fermi points

$$
R_{a, b}^{(\eta)}(\omega)=-i \int_{-\infty}^{+\infty} d t e^{i \omega t}\left\langle T O_{a}^{(\eta)}(t) O_{b}^{(\eta) \dagger}(0)\right\rangle
$$

with $(\eta)=(S S)$ and $(T S)$.

Due to the nontrivial structure of the Fermi line, the symmetry of the pair wave function is obtained by diagonalizing the matrices $R_{a, b}^{(S S)}$ and $R_{a, b}^{(T S)}$ in the low-energy limit and looking for the eigenvector corresponding to the largest eigenvalue. We recall at this point that the derivatives with respect to the frequency of the response functions, $\bar{R}^{(\eta)}$ $=\partial R^{(\eta)} / \partial \log (\omega)$, have well-defined scaling properties. ${ }^{26}$ In the case of the superconducting response functions, the scaling equations read

$$
\begin{aligned}
\frac{\partial \bar{R}_{a, b}^{(S S)}(\omega)}{\partial \log (\Lambda)}= & \frac{1}{\pi v_{a}} \sum_{c}\left[\left(1-\delta_{a, c}\right)\left(c_{a, c}^{(+)}+c_{a, c}^{(-)}\right)\right. \\
& \left.+\delta_{a, c}\left(f_{a,-c}^{(+)}+f_{a,-c}^{(-)}\right)\right] \bar{R}_{c, b}^{(S S)}(\omega),
\end{aligned}
$$

$$
\begin{aligned}
\frac{\partial \bar{R}_{a, b}^{(T S)}(\omega)}{\partial \log (\Lambda)}= & \frac{1}{\pi v_{a}} \sum_{c}\left[\left(1-\delta_{a, c}\right)\left(c_{a, c}^{(+)}-c_{a, c}^{(-)}\right)\right. \\
& \left.+\delta_{a, c}\left(f_{a,-c}^{(+)}-f_{a,-c}^{(-)}\right)\right] \bar{R}_{c, b}^{(T S)}(\omega) .
\end{aligned}
$$

Because of the symmetry of the Fermi line, the eigenvectors of $R_{a, b}$ transform according to the irreducible representations of $C_{4 v}$.

In the case of density-wave response functions, given a momentum transfer $\mathbf{q}$ across the Fermi line connecting the Fermi points $(a, \bar{b})$, the only other pair connected by $\mathbf{q}$ is made of the inverted points $(-\bar{b},-a)$. We may form, therefore, two different operators for the same momentum $\mathbf{q}$

$O_{1}^{(C D W / S D W)}(\mathbf{q})=\int d p\left[\psi_{a \uparrow}^{\dagger}(p+q) \psi_{\bar{b} \uparrow}(p) \pm \psi_{a \downarrow}^{\dagger}(p+q) \psi_{\bar{b} \downarrow}(p)\right]$,

$$
\begin{aligned}
O_{2}^{(C D W / S D W)}(\mathbf{q})= & \int d p\left[\psi_{-\bar{b} \uparrow}^{\dagger}(p+q) \psi_{-a \uparrow}(p)\right. \\
& \left. \pm \psi_{-\bar{b} \downarrow}^{\dagger}(p+q) \psi_{-a \downarrow}(p)\right],
\end{aligned}
$$

which give rise to two different response functions

$$
\begin{aligned}
& R_{1,1}^{(\eta)}(\mathbf{q}, \omega)=-i \int_{-\infty}^{+\infty} d t e^{i \omega t}\left\langle T O_{1}^{(\eta)}(\mathbf{q}, t) O_{1}^{(\eta) \dagger}(\mathbf{q}, 0)\right\rangle, \\
& R_{1,2}^{(\eta)}(\mathbf{q}, \omega)=-i \int_{-\infty}^{+\infty} d t e^{i \omega t}\left\langle T O_{1}^{(\eta)}(\mathbf{q}, t) O_{2}^{(\eta) \dagger}(\mathbf{q}, 0)\right\rangle .
\end{aligned}
$$

The response functions $R_{1,1}^{(\eta)}(\mathbf{q}, \omega)$ and $R_{1,2}^{(\eta)}(\mathbf{q}, \omega)$ are actually entangled by the interactions, as can be seen from the scaling equations

$$
\begin{aligned}
\frac{\partial \bar{R}_{1,1}^{(C D W)}(\mathbf{q}, \omega)}{\partial \log (\Lambda)}= & \frac{2}{\pi\left(v_{a}+v_{b}\right)}\left[\left(c_{a,-\bar{b}}^{(+)}-2 c_{a,-\bar{b}}^{(-)}\right) \bar{R}_{1,2}^{(C D W)}(\mathbf{q}, \omega)\right. \\
& +\left(f_{a, \bar{b}}^{(+)}-2 f_{a, \bar{b}}^{(-)} \bar{R}_{1,1}^{(C D W)}(\mathbf{q}, \omega)\right] \\
\frac{\partial \bar{R}_{1,2}^{(C D W)}(\mathbf{q}, \omega)}{\partial \log (\Lambda)}= & \frac{2}{\pi\left(v_{a}+v_{b}\right)}\left[\left(c_{a,-\bar{b}}^{(+)}-2 c_{a,-\bar{b}}^{(-)}\right) \bar{R}_{1,1}^{(C D W)}(\mathbf{q}, \omega)\right. \\
& +\left(f_{a, \bar{b}}^{(+)}-2 f_{a, \bar{b}}^{(-)} \bar{R}_{1,2}^{(C D W)}(\mathbf{q}, \omega)\right] \\
\frac{\partial \bar{R}_{1,1}^{(S D W)}(\mathbf{q}, \omega)}{\partial \log (\Lambda)}= & \frac{2}{\pi\left(v_{a}+v_{b}\right)}\left[c_{a,-\bar{b}}^{(+)} \bar{R}_{1,2}^{(S D W)}(\mathbf{q}, \omega)\right. \\
& \left.+f_{a, \bar{b}}^{(+)} \bar{R}_{1,1}^{(S D W)}(\mathbf{q}, \omega)\right] \\
\frac{\partial \bar{R}_{1,2}^{(S D W)}(\mathbf{q}, \omega)}{\partial \log (\Lambda)}= & \frac{2}{\pi\left(v_{a}+v_{b}\right)}\left[c_{a,-\bar{b}}^{(+)} \bar{R}_{1,1}^{(S D W)}(\mathbf{q}, \omega)\right. \\
& \left.+f_{a, b}^{(+)} \bar{R}_{1,2}^{(S D W)}(\mathbf{q}, \omega)\right] .
\end{aligned}
$$




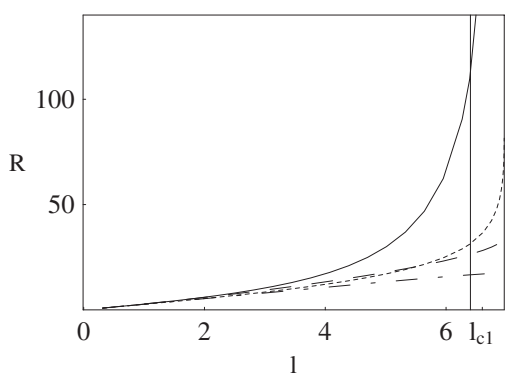

FIG. 4. Plot of the dominant response functions for each different kind of order as a function of $l=-\log \left(\Lambda / E_{c}\right)$. From top to bottom, we have SS $d_{x^{2}-y^{2}}$ (solid line), CDW (dotted line), SDW (dashed line), and TS (dotted-dashed line) response functions. The data correspond to a Hubbard model with $N=48, t^{\prime}=-0.2 t$, and $U$ $=t$, at the Van Hove filling. The point where the renormalization group flow diverges is at $l^{*} \approx 6.96$. The vertical line at $l_{c 1} \approx 6.40$ indicates the energy scale at which the Fermi line starts to collapse.

In the charge-density-wave and spin-density-wave sectors, the symmetry of the dominant correlations is determined by finding the largest eigenvalue from the diagonalization of the $2 \times 2$ blocks

$$
\left(\begin{array}{ll}
R_{1,1}^{(\eta)}(\mathbf{q}, \omega) & R_{1,2}^{(\eta)}(\mathbf{q}, \omega) \\
R_{1,2}^{(\eta)}(\mathbf{q}, \omega) & R_{1,1}^{(\eta)}(\mathbf{q}, \omega)
\end{array}\right)
$$

for $\eta=\mathrm{CDW}$ and SDW.

We have plotted in Figs. 4 and 5 the evolution of the response functions for arrays with $N=48$ and 76, respectively, taking as a representative the largest eigenvalue for each kind of order, i.e., SS, TS, CDW, and SDW. The dominant instability is found in the superconducting channel with $d_{x^{2}-y^{2}}$ symmetry. The corresponding eigenvector of the matrix $R_{a, b}^{(S S)}$ represents the amplitude of the Cooper-pair wave function along the Fermi line, which turns out to be peaked (with opposite signs) at the two hot spots around $(\pi, 0)$ and $(0, \pi)$, and vanishing elsewhere. This result is consistent with the dominance of the $d$-wave correlations observed in $N$-leg Hubbard ladders for small values of $N$.

At this point, we can go beyond the renormalization group solution of the model by incorporating the nonperturbative methods used in the previous section for the analysis of the quasiparticle properties. Our approach consists in computing the string of anomalous scaling dimensions $\left\{\gamma_{a}^{(F S)}\right\}$ obtained

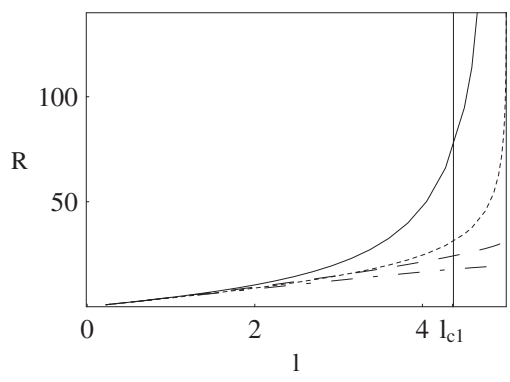

FIG. 5. Similar plot as in Fig. 4 for $N=76$, with $l^{*} \approx 4.99$ and $l_{c 1} \approx 4.36$. from the forward-scattering couplings $f_{a, b}^{(+)}, f_{\bar{a}, \bar{b}}^{(+)}, f_{a, \bar{b}}^{(+)}$, and $f_{\bar{a}, b}^{(+)}$ at each renormalization group step. To this end, we use the couplings calculated by solving the renormalization group equations to diagonalize the Hamiltonian in Eq. (8) for 0 $<l<l^{*}$. On technical grounds, this amounts to the bosonization of the electron system at different values of $\Lambda$. We end up in this way with an anomalous electron dimension $\gamma_{a}^{(F S)}(l)$ that depends on the energy scale. The numerical computation of $\gamma_{a}^{(F S)}(l)$ reveals that, at a scale $l_{c 1}<l^{*}$, the anomalous dimension for the Fermi points nearest to $(\pi, 0)$ and $(0, \pi)$ diverges (namely, for $k_{a}=\pi / N$ and $\left.k_{a}=\pi-\pi / N\right)$, while $\gamma_{a}^{(F S)}\left(l_{c 1}\right)$ remains small for the rest of the electron fields.

The divergence of the anomalous dimension $\gamma_{a}^{(F S)}(l)$ near $(\pi, 0)$ and $(0, \pi)$ can be traced back to the singular behavior of the change of variables (15) and (16) at the point $l=l_{c 1}$. The corresponding operators $B_{a}^{\dagger}(q)$ and $B_{\bar{a}}(q)$ have projections $\widetilde{s}_{a b}, \widetilde{s}_{\bar{a} \bar{b}}, \tilde{t}_{a \bar{b}}$, and $\widetilde{t}_{\bar{a} b}$ that diverge for particular components $\widetilde{B}_{b}^{\dagger}(q)$ and $\widetilde{B}_{b}^{-}(q)$. These are made of a $C_{4 v}$-symmetric combination of operators peaked at the points nearest to $(\pi, 0)$ and $(0, \pi)$. When rewritten in terms of the density modes $B_{a}^{\dagger}(q)+B_{\bar{a}}(-q)$, the change of variables leads, however, to a projection $\widetilde{s}_{a b}+\widetilde{t}_{\bar{a} b}\left(=\widetilde{s}_{\bar{a} \bar{b}}+\widetilde{t}_{a \bar{b}}\right)$ that vanishes at $l=l_{c 1}$ for the mentioned component. This shows that the situation is similar to that found in a one-dimensional (1D) system with two subbands, where under certain conditions the Luttinger liquid parameter $K_{+}$in the channel of the total charge density may vanish at a certain point of the renormalization group flow. ${ }^{32}$ The correspondence is such that $\widetilde{s}_{a b}$ $\sim\left(\sqrt{K_{+}}+1 / \sqrt{K_{+}}\right) / 2 \sqrt{2}$ and $\tilde{t}_{\bar{a} b} \sim\left(\sqrt{K_{+}}-1 / \sqrt{K_{+}}\right) / 2 \sqrt{2}$. Specifically, in the model of two subbands with equal Fermi velocity $v_{a}=v_{b}=v_{F}$, the Luttinger liquid parameters in the respective channels for the symmetric and antisymmetric combination of the subbands are

$$
K_{ \pm}=\sqrt{\frac{\pi v_{F}+\left(f_{a, a}^{(+)} \pm f_{a, b}^{(+)}\right)-\left(f_{a, \bar{a}}^{(+)} \pm f_{a, \bar{b}}^{(+)}\right)}{\pi v_{F}+\left(f_{a, a}^{(+)} \pm f_{a, b}^{(+)}\right)+\left(f_{a, \bar{a}}^{(+)} \pm f_{a, b}^{(+)}\right)}} .
$$

We have checked that, as well as in the system of two subbands, the vanishing of $\widetilde{s}_{a b}+\widetilde{t}_{\bar{a} b}$ takes place in our model when the interband interactions $f_{a, b}^{(+)}$between the points nearest to $(\pi, 0)$ and $(0, \pi)$ grow large enough upon renormalization to drive to zero the corresponding Luttinger liquid parameter.

We have then identified the origin of the singular behavior in the change of variables (15) and (16). This takes place when the mentioned couplings $f_{a, b}^{(+)}$become of the order of the Fermi velocity $v_{a}$, that is, when they are leaving the weak-coupling regime. Thus, the divergence in the anomalous scaling dimension at $l=l_{c 1}$ is found before entering the regime of large growth of the renormalized couplings, and it may be considered a more reliable result than the predictions obtained from the large growth of the response functions.

In order to clarify the physical meaning of the divergence of $\gamma_{a}^{(F S)}$, we recall that this is actually part of the full anoma- 

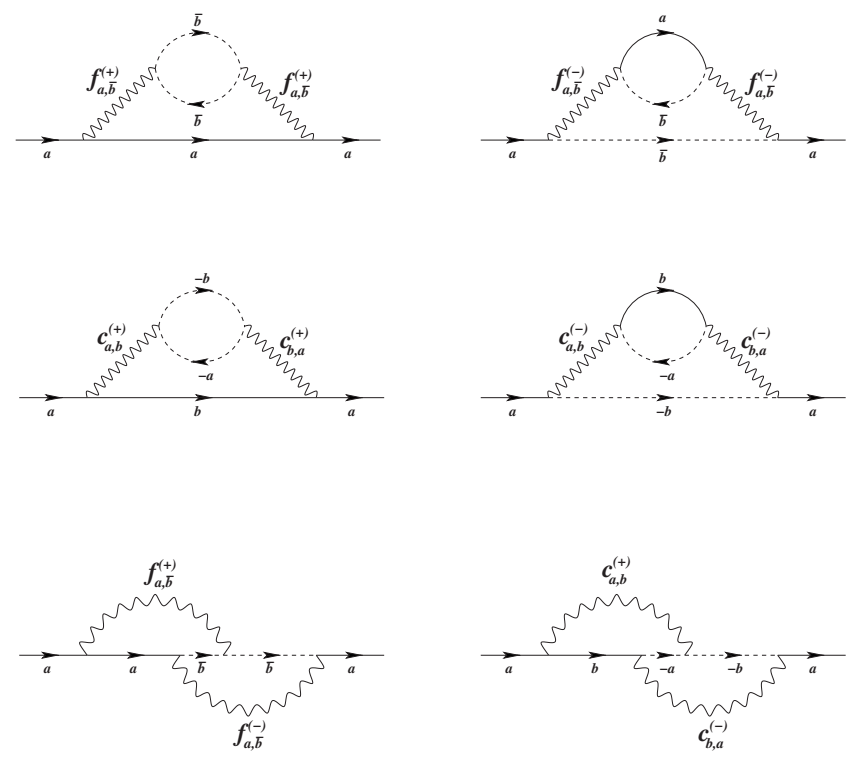

FIG. 6. Diagrams contributing to the electron self-energy which renormalize to lowest order the electron quasiparticle weight.

lous dimension $\gamma_{a}$ of the electron field operator $\psi_{a \sigma}$. In correspondence with Eq. (17), the anomalous dimension $\gamma_{a}$ can also be defined from the time dependence of the electron propagator

$$
\left\langle\psi_{a \sigma}^{\dagger}(x, t) \psi_{a \sigma}\left(x, t^{\prime}\right)\right\rangle \sim \frac{1}{\left|t-t^{\prime}\right|^{1+\gamma_{a}}} .
$$

A nonvanishing anomalous dimension is detected, in general, from the renormalization of the electron wave function at low energies. This is usually computed from inspection of the self-energy corrections that depend on the electron frequency, which have a perturbative expansion in our model starting with the contributions shown in Fig. 6. It is clear that, with our nonperturbative bosonization approach, we have already captured the dependence obtained from the sum of all the diagrams made of forward-scattering couplings $f_{a, b}^{(+)}, f_{a, \bar{b}}^{(+)}$. From the diagrams in Fig. 6, one can obtain the first perturbative orders of the full anomalous dimension

$$
\begin{aligned}
\gamma_{a}= & \sum_{b} \frac{\alpha}{\left(v_{a}+v_{b}\right)^{2}}\left[\left(f_{a, b}^{(+)}\right)^{2}+\left(f_{a, b}^{(-)}\right)^{2}-f_{a, b}^{(+)} f_{a, b}^{(-)}\right] \\
& +\sum_{b} \frac{\alpha^{\prime}}{\left(v_{a}+v_{b}\right)^{2}}\left[\left(c_{a, b}^{(+)}\right)^{2}+\left(c_{a, b}^{(-)}\right)^{2}-c_{a, b}^{(+)} c_{a, b}^{(-)}\right]+\cdots .
\end{aligned}
$$

The terms depending only on forward-scattering couplings in Eq. (41) are nothing but the expansion of

$$
\gamma_{a}^{(F S)}=\frac{1}{2} \sum_{b}\left(\left|\tilde{s}_{a b}\right|^{2}+\left|\tilde{t}_{a b}\right|^{2}\right)-\frac{1}{2}
$$

as illustrated, for instance, in the system of the two subbands by the expansion of $\gamma_{a}^{(F S)}=(1 / 8)\left(K_{+}+1 / K_{+}+K_{-}+1 / K_{-}-4\right)$. It then becomes clear that, once a zero is reached in one of the Luttinger liquid parameters, the resulting divergence is translated to the full anomalous dimension $\gamma_{a}$ and cannot be cured by the rest of the terms in the expansion. We remark that the terms in Eq. (41) made of just the backscattering and Cooper-pair couplings must sum to a positive definite quantity, as they alone define a consistent field theory. Moreover, the terms that mix the forward-scattering couplings with backscattering and Cooper-pair couplings are partially taken into account in our approach, as the computation of $\gamma_{a}^{(F S)}(l)$ with the renormalized couplings already incorporates the dressing of the forward-scattering vertices with the interactions integrated out in the renormalization process. Finally, the most compelling evidence for the divergence of the full anomalous dimension is that the singularity in $\gamma_{a}^{(F S)}$ arises actually from a branch cut in the space of couplings, implying necessarily that the nonanalytic behavior is transmitted to the anomalous dimension $\gamma_{a}$.

From the physical point of view, the divergence of $\gamma_{a}$ is the reflection of a local instability in the string of Fermi points, since at this stage it only affects the points nearest to $(\pi, 0)$ and $(0, \pi)$. We can first analyze the effect of the divergence of $\gamma_{a}$ by recalling that this anomalous dimension dictates the behavior of the observables defined from the electron propagator. In particular, the density of states, which we define here as a quantity $n_{a}(\varepsilon)$ depending on the Fermi point, is obtained by taking the Fourier transform of the time dependence of the electron propagator (40). Thus, we get the power-law behavior

$$
n_{a}(\varepsilon) \sim \varepsilon^{\gamma_{a}},
$$

where the energy is measured in units of the high-energy cutoff. Very large values of the exponent $\gamma_{a}$ indicate a very strong suppression of the electron quasiparticles, as the shape of the density of states becomes almost flat at low energies. We see that, at the point where $\gamma_{a}$ diverges, a gap opens effectively in the spectrum of fermion excitations, since the density of states vanishes then over a range of low energies.

The opening of a gap near $(\pi, 0)$ and $(0, \pi)$ in the string of Fermi points can be studied more precisely in our renormalization group framework. For this purpose, we deal with the angle-resolved quasiparticle weight $Z_{a}$, which we relate in our approach to the anomalous dimension $\gamma_{a}^{(F S)}(l)$ through the scaling equation

$$
\frac{1}{Z_{a}(l)} \frac{\partial Z_{a}(l)}{\partial l}=-\gamma_{a}^{(F S)}(l) .
$$

We have integrated numerically Eq. (44) for every $a$ in the range $0<l<l_{c 1}$. The result is shown in Fig. 7 (top line) for $N=48$. We observe that the quasiparticle weight vanishes at $l=l_{c 1}$ for the Fermi points nearest to $(\pi, 0)$ and $(0, \pi)$, while in the rest of the Fermi line the renormalization is quite mild. We remark that the vanishing of $Z_{a}$ indicates the disappearance of fermion excitations at the Fermi point $a$ below an energy given by $\Lambda_{c 1}=E_{c} e^{-l_{c 1}}$. In this respect, the physical effect is different such that in a Luttinger liquid, where the anomalous dimension of the electron is independent of the energy scale. A constant value of $\gamma_{a}$ implies a quasiparticle weight $Z_{a} \sim \exp \left(-l \gamma_{a}\right)$, vanishing only at zero energy. In our 


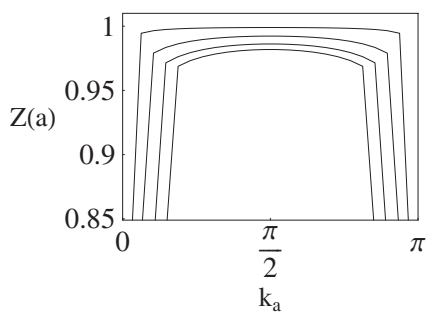

FIG. 7. Angle-resolved quasiparticle weight $Z_{a}$ as a function of the transverse momentum $k_{a}$ for the first four iterations of the combined renormalization group and bosonization approaches for an array with $N=48$. From top to bottom, $Z_{a}$ is shown at the points $l_{c 1}$, $l_{c 2}, l_{c 3}$, and $l_{c 4}$, where a new set of Fermi points decouples from the spectrum. The parameters are the same as in Fig. 4.

case, however, the quasiparticle weight vanishes at an energy $\Lambda_{c 1} \neq 0$, implying the opening of a gap right below that energy scale at the points near $(\pi, 0)$ and $(0, \pi)$.

It is interesting to follow the trend of the rest of the Fermi points by further lowering the energy scale. This is achieved by solving again the renormalization group equations for the set of Fermi points obtained by excluding the decoupled ones. For $N=48$, we remain with $96-8=88$ Fermi points, and the new initial conditions are set by evaluating the corresponding couplings at $l_{c 1}$, as obtained from the previous renormalization group flow. According to the above discussion, all of them are still in the weak-coupling regime, making the calculation well posed. The new renormalization group flow is obtained numerically starting from $l_{c 1}$. Again, an instability is observed, due to the growth of the interactions involving scattering between Fermi points with smaller Fermi velocity (i.e., at $k_{a}=3 \pi / N$ and $k_{a}=\pi-3 \pi / N$ ). Following the same procedure shown above, the new anomalous dimensions $\gamma_{a}$ and the quasiparticle weights $Z_{a}$ are computed. Also in this case, the $\gamma_{a}$ for the Fermi points nearest to the hot spots diverge at $l_{c 2}>l_{c 1}$. The new quasiparticle weights are obtained by solving Eq. (44) in the range $\left(l_{c 1}, l_{c 2}\right)$, with initial conditions dictated by the previous integration of the same equations. In Fig. 7 (second line from top), we see that $Z_{a}$ vanishes again only for the points with smaller Fermi velocity. We observe that its renormalization is now more pronounced at different angles with respect to the previous stage.

This procedure can be iterated, giving rise to the progressive destruction of the Fermi line, from $(\pi, 0)$ and $(0, \pi)$ toward the diagonals of the Brillouin zone. It should be noted that the energy scale $\Lambda_{c n}=E_{c} e^{-l_{c n}}$ at which the $n$th Fermi point disappears may become very small. In Fig. 7, we have plotted the angular distribution of $Z_{a}$ for the first four iterations, corresponding to the destruction of the Fermi points having transverse momentum $\pm(2 n+1) \pi / 48$ and $\pm \pi \mp(2 n$ $+1) \pi / 48$, with $n=0,1,2$, and 3 . We observe that, by increasing the number of iterations, the renormalization of $Z_{a}$ away from the hot spots becomes more and more pronounced. The plot is actually reminiscent of the results for the quasiparticle renormalization shown in Ref. 20. There is a clear indication that, while the collapse occurs on finite arcs around $(\pi, 0)$ and $(0, \pi)$, the rest of the Fermi line results renormalized by the interactions.

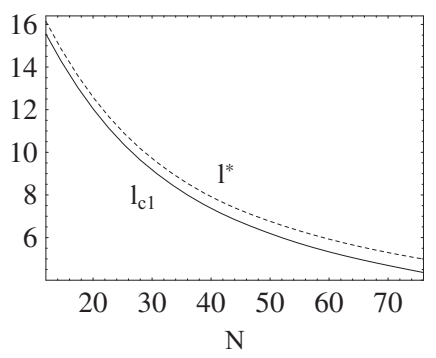

FIG. 8. Plot of the critical scales $l^{*}$ (dotted line) and $l_{c 1}$ (full line) as a function of $N$. The parameters are the same as in Fig. 4.

The comparison of the plots in Figs. 4 and 5 gives a fair idea about the dependence of the above results on the size of the array of chains. The $d$-wave superconducting correlations turn out to be always dominant (see Fig. 5), but the collapse of the Fermi line at the hot spots prevents them from growing large in any case. In Fig. 8, we have represented the trend of the critical scales $l^{*}$ and $l_{c 1}$ as a function of $N$. It appears that $l^{*}$ approaches an asymptotic value in the large- $N$ limit, while the difference between $l^{*}$ and $l_{c 1}$ does not vanish by increasing the size of the array. This suggests that the development of the pseudogap should survive in the 2D limit, ending up with a paradigm of electron liquid in which the Fermi surface is progressively destroyed as the system is probed at lower energy scales.

\section{CONCLUSION}

In this paper, we have studied the low-energy electronic instabilities in a 2D Hubbard model formed by a large array of coupled chains. Within a low-energy cutoff $E_{c}$ about the Fermi level, the different interactions can be classified into a reduced number of channels, ${ }^{10}$ which coincide essentially with those obtained from the analysis of the kinematics for a 2D Fermi surface. ${ }^{30}$ In the model with bare on-site repulsion $U$, the different couplings in momentum space have a strength of the order of $U / N$, which makes appropriate the use of renormalization group methods to determine the scaling of the couplings as the cutoff is reduced toward the Fermi level.

Complementing this approach, we have also applied bosonization methods to describe the dressing of the electron fields at the different Fermi points. Thus, we have computed the string of anomalous scaling dimensions $\left\{\gamma_{a}\right\}$ with the effective couplings at each renormalization group step. This combines the power of the renormalization group and the bosonization methods, allowing to characterize the points where the metallic phase may break down. In this way, we have been able to discern the competition between the tendency to the formation of ordered phases in the $2 \mathrm{D}$ electron liquid and the charge instabilities that modify the Fermi line.

Our main interest has been focused on the instabilities that appear when the Fermi level is close to the Van Hove singularity arising from the saddle points at $(\pi, 0)$ and $(0, \pi)$. It is well known that the singularity in the density of states leads to strong correlations that signal the tendency toward a spin-density-wave phase or $d$-wave superconductivity at low 
energies. This has been studied in the past using mainly renormalization group methods. An important drawback in this approach comes, however, from the unconventional form of the $2 \mathrm{D}$ perturbative expansion, with terms that diverge at low energies as the square of $\log (\varepsilon)$ and which are not, therefore, properly renormalizable.

It has been actually pointed out that the renormalization of the quasiparticle properties has to be quite significant near the Van Hove singularity, and that this may lead to a drastic softening of the electron correlations. ${ }^{20}$ In our approach, we have recovered some of the trends found within the conventional renormalization group schemes, like the strong renormalization of the dispersion relation around the saddle points ${ }^{15,23}$ or the growth of the $d$-wave superconducting correlations. ${ }^{11,12,14,16-19,21,22}$ Our main finding is anyhow that, at the Van Hove filling, the progressive destruction of the Fermi line takes place around the saddle points before any correlation may diverge in the low-energy limit. This is a sensible result in our model, as the 1D renormalization group approach is well defined, while the analysis of the breakdown of the metallic phase is carried out with a nonperturbative bosonization approach.

This study may also have implications for the understanding of the pseudogap phase in the copper-oxide superconductors. The loss of fermion excitations takes place in our model as the electron fields are attenuated due to the dressing from the renormalized interactions at the hot spots around $(\pi, 0)$ and $(0, \pi)$. This happens as the charge stiffness goes to zero at some of the Fermi points in the low-energy limit. We stress that this behavior marks the approach of a critical point, characterized by the vanishing of some of the Luttinger liquid parameters encoded in the expression (20) for the anomalous dimension. ${ }^{32,33}$ The critical point is reached as some of the effective couplings leave the weak-coupling regime, so our description of the pseudogap does not rely on strong-coupling features to account for the destruction of the Fermi line. In future studies, it would be interesting to investigate the extrapolation of the present results to systems with larger number of chains. It seems plausible that the destruction of the Fermi line we have described may persist for increasing number of chains, as it relies on a critical behavior that is a robust property of the bosonization of the system in the low-energy limit.

\section{ACKNOWLEDGMENTS}

The financial support of the Ministerio de Educación y Ciencia (Spain) through Grant No. FIS2005-05478-C02-02 is gratefully acknowledged. E.P. was also supported by Consorzio Nazionale Interuniversitario per le Scienze Fisiche della Materia.
${ }^{1}$ For a review, see, for instance, T. Timusk and B. Statt, Rep. Prog. Phys. 62, 61 (1999).

${ }^{2}$ H. Ding, T. Yokoya, J. C. Campuzano, T. Takahashi, M. Randeria, M. R. Norman, T. Mochiku, K. Kadowaki, and J. Giapintzakis, Nature (London) 382, 51 (1996); A. G. Loeser, Z.-X. Shen, D. S. Dessau, D. S. Marshall, C. H. Park, P. Fournier, and A. Kapitulnik, Science 273, 325 (1996).

${ }^{3}$ M. R. Norman, H. Ding, M. Randeria, J. C. Campuzano, T. Yokoya, T. Takeuchi, T. Takahashi, T. Mochiku, K. Kadowaki, P. Guptasarma, and D. G. Hinks, Nature (London) 392, 157 (1998).

${ }^{4}$ B. Janko, J. Maly, and K. Levin, Phys. Rev. B 56, R11407 (1997).

${ }^{5}$ J. Schmalian, D. Pines, and B. Stojkovic, Phys. Rev. Lett. 80, 3839 (1998).

${ }^{6}$ C. Castellani, C. Di Castro, and M. Grilli, Phys. Rev. Lett. 75, 4650 (1995).

${ }^{7}$ S. Chakravarty, R. B. Laughlin, D. K. Morr, and C. Nayak, Phys. Rev. B 63, 094503 (2001).

${ }^{8}$ For a recent account, see P. W. Anderson, Phys. Rev. Lett. 96, 017001 (2006).

${ }^{9}$ C. M. Varma, Phys. Rev. B 73, 155113 (2006).

${ }^{10}$ H.-H. Lin, L. Balents, and M. P. A. Fisher, Phys. Rev. B 56, 6569 (1997).

${ }^{11}$ H. J. Schulz, Europhys. Lett. 4, 609 (1987); P. Lederer, G. Montambaux, and D. Poilblanc, J. Phys. (Paris) 48, 1613 (1987); J. E. Dzyaloshinskii, Pis'ma Zh. Eksp. Teor. Fiz. 46, 97 (1987) [JETP Lett. 46, 118 (1987)].

${ }^{12}$ J. González, F. Guinea, and M. A. H. Vozmediano, Europhys.
Lett. 34, 711 (1996).

${ }^{13}$ L. B. Ioffe and A. J. Millis, Phys. Rev. B 54, 3645 (1996).

${ }^{14}$ D. Zanchi and H. J. Schulz, Phys. Rev. B 54, 9509 (1996).

${ }^{15}$ J. González, F. Guinea, and M. A. H. Vozmediano, Nucl. Phys. B 485, 694 (1997).

${ }^{16}$ J. V. Alvarez, J. González, F. Guinea, and M. A. H. Vozmediano, J. Phys. Soc. Jpn. 67, 1868 (1998).

${ }^{17}$ C. J. Halboth and W. Metzner, Phys. Rev. B 61, 7364 (2000); Phys. Rev. Lett. 85, 5162 (2000).

${ }^{18}$ J. González, F. Guinea, and M. A. H. Vozmediano, Phys. Rev. Lett. 84, 4930 (2000).

${ }^{19}$ C. Honerkamp, M. Salmhofer, N. Furukawa, and T. M. Rice, Phys. Rev. B 63, 035109 (2001).

${ }^{20}$ D. Zanchi, Europhys. Lett. 55, 376 (2001).

${ }^{21}$ B. Binz, D. Baeriswyl, and B. Douçot, Eur. Phys. J. B 25, 69 (2002).

${ }^{22}$ A. A. Katanin and A. P. Kampf, Phys. Rev. B 68, 195101 (2003).

${ }^{23}$ A. A. Katanin and A. P. Kampf, Phys. Rev. Lett. 93, 106406 (2004).

${ }^{24}$ This set of low-energy couplings becomes completely equivalent to that introduced in Ref. 10 after assuming the spin independence of the interactions in every channel.

${ }^{25}$ For even $N$, the set of couplings in the Hamiltonian (5) has to be, in principle, completed with a number of vertical umklapp processes (direct and exchange), in which the transverse momentum $k_{a}$ is conserved modulus $2 \pi$ (see Ref. 10). We have checked by numerical inspection that their couplings remain small under the renormalization group flow and that they do not affect the flow of the rest of the couplings so, for the sake of simplicity, we 
have not included them explicitly in the general discussion.

${ }^{26}$ J. Sólyom, Adv. Phys. 28, 201 (1979).

${ }^{27}$ This behavior has also been found, in the context of a model with constant interaction potential between Luttinger chains, by L. Bartosch and P. Kopietz, Phys. Rev. B 55, 15360 (1997); see also H. J. Schulz, ibid. 53, R2959 (1996).

${ }^{28}$ S. Ledowski and P. Kopietz, J. Phys.: Condens. Matter 15, 4779 (2003).

${ }^{29}$ S. Ledowski, P. Kopietz, and A. Ferraz, Phys. Rev. B 71, 235106
(2005).

${ }^{30}$ R. Shankar, Rev. Mod. Phys. 66, 129 (1994).

${ }^{31}$ An equivalent set of equations for a different choice of independent couplings can be found in Ref. 10.

${ }^{32}$ J. V. Alvarez and J. González, Phys. Rev. Lett. 91, 076401 (2003).

${ }^{33}$ This behavior has also been observed in systems with several 1D subbands at the Fermi level, see, for instance, J. González and E. Perfetto, Phys. Rev. B 72, 205406 (2005) 